\title{
Avaliação da eficácia de produtos isoterápicos no controle de Rhipicephalus (Boophilus) microplus
}

\section{Evaluation of the efficacy of products isoterapicos in control of Rhipicephalus (Boophilus) microplus}

\author{
Tânia Regina Bettin dos Santos ${ }^{1 *}$, Cíntia Lidiane Guidotti Aguiar², Luciana Prestes', \\ Marina Oliveira Daneluz ${ }^{3}$, Bruna Farias Alves ${ }^{3}$, Maria Izabel de Tourinho e Torres ${ }^{3}$
}

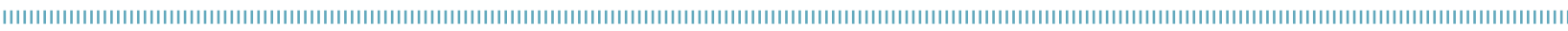

RESUMO: O Rhipicephalus (Boophilus) microplus é responsável por consideráveis perdas na pecuária brasileira. $\mathrm{O}$ controle desse parasita tem sido feito, principalmente, com o uso de carrapaticidas, sendo os isoterápicos uma alternativa para seu combate. Objetivou-se neste trabalho analisar a eficiência da utilização da isopatia como ferramenta de controle alternativo do carrapato-do-boi. Foram utilizadas 18 fêmeas bovinas de raça europeia, mantidas em sistema de pecuária extensiva, no município de Canguçu, Rio Grande do Sul. Os animais foram divididos em grupo controle (Lote A) e grupo tratado com isoterápico (Lote B). O sal mineral foi utilizado como veículo para a administraçáo da isopatia, sendo que o Lote A recebeu sal mineral com placebo (açúcar) e o Lote B sal mineral com isopatia. Os animais foram infestados artificialmente com 10 mil larvas de $R$. (B.) microplus, e no $20^{\circ}, 21^{\circ}$ e $22^{\circ}$ dias pós-infestação, foram realizadas contagem e coleta de fêmeas ingurgitadas. As teleóginas de cada lote foram divididas ao acaso em nove grupos de dez indivíduos para análise dos parâmetros biológicos e posterior cálculo do Índice Nutricional e Índice de Eficácia Reprodutiva. Para comparação da média de teleóginas de cada grupo, foi empregada análise de variância e Teste LSD. Os bovinos tratados com isopatia apresentaram uma reduçáo de $53,4 \%$ do número médio de teleóginas, quando comparados com o grupo náo tratado $(\mathrm{p}=0,001)$. Quanto aos padróes biológicos, não houve diferença estatística. Conclui-se que o uso de isopatia foi capaz de reduzir a infestaçáo de $R$. (B.) microplus, mostrando-se uma alternativa promissora para o controle deste ixodídeo.

PALAVRAS-CHAVE: Materia medica; homeopatia; bioterápicos.

\begin{abstract}
The Rhipicephalus (Boophilus) microplus is responsible for considerable losses in Brazilian cattle. The control of such parasites has been done mainly with the use of acaricides, and the isopathy is an alternative. The objective of this work is to analyze the efficiency of the use of isopathy as an alternative tool to control the cattle tick. We used 18 cows of the European race, kept in extensive livestock system in the municipality of Canguçu, Rio Grande do Sul, Brazil. The animals were divided into control group (Lot A) and group treated with isopathy (Lot B). Mineral salt was used as a vehicle for the administration of isopathy, Lot A received mineral salt with placebo (sugar) and Lot B received mineral salt with isopathy. The animals were artificially infested with 10 thousan larvae of $R$. (B.) microplus, and in the days 20, 21 and 22 post-infestation engorged females were counted and collected. The ticks from each batch were randomly divided into nine groups of ten subjects each for analysis of biological parameters and subsequent calculation of the Nutritional Index and the Effectiveness Reproductive Index. For comparison of average engorged females from each group were employed ANOVA and LSD Test. The animals treated with isopathy showed a $53.4 \%$ reduction in the average number of ticks when compared with the untreated group ( $\mathrm{p}=0.001)$. As for biological standards, there was no statistical difference. It is concluded that the use of isopathy was able to reduce infestation of $R$. (B.) microplus, showing up as a promising alternative to control this ticks.
\end{abstract}

KEYWORDS: Materia medica; homeopathy; biotherapics.

'Faculdade de Veterinária, Departamento de Medicina Veterinária Preventiva, Laboratório de Doenças Parasitárias (Ladopar), Universidade Federal de Pelotas (UFPel) - Pelotas (RS), Brasil.

${ }^{2}$ Instituto de Biologia, Departamento de Microbiologia e Parasitologia, UFPel - Pelotas (RS), Brasil.

${ }^{3}$ Laboratório de Doenças Parasitárias, Faculdade de Veterinária UFPel - Pelotas (RS), Brasil.

*Autor correspondente: trb.santos@hotmail.com

Recebido em: 13/11/2013. Aceito em: 28/10/2015 


\section{INTRODUÇÃO}

Rhipicephalus (Boophilus) microplus, popularmente conhecido como carrapato-do-boi, tem sido o grande responsável por consideráveis perdas nos sistemas de pecuária no Brasil, tanto pelas lesões no couro, queda do desempenho e transmissão de doenças, quanto pelos custos com tratamentos que, muitas vezes, tornam-se ineficazes (FArIas, 2007). Além dos prejuízos diretos, decorrentes da diminuiçáo de desempenho pelo hematofagismo e desvalorização do couro, as perdas são agravadas pela transmissão dos hemoparasitos Babesia bovis, Babesia bigemina e Anaplasma marginale, causadores da tristeza parasitária bovina, e pelos altos gastos com insumos (Grisi et al., 2002).

O controle desse parasita tem sido feito, principalmente, com o uso de carrapaticidas (PAIm et al., 2011; Olivo et al., 2013; Silva Filho et al., 2013). Porém, o uso inadequado e desenfreado desses químicos tem selecionado populaçóes de carrapatos resistentes a inúmeros princípios ativos.

A utilização de métodos alternativos para controlar o carrapato tem sido discutida com a finalidade de melhorar as condiçôes dos sistemas de produção de corte e demonstrar aos produtores algumas ferramentas aliadas, como por exemplo, a isopatia.

Os isoterápicos são produzidos a partir do agente causador da doença ou do desequilíbrio (Silva et al. 2007). Sáo produtos cujo insumo ativo poderá ser de origem endógena ou exógena (alérgenos, alimentos, cosméticos, medicamentos, toxinas, parasitos e outros). O método que utiliza isoterápicos como tratamento é chamado isopatia: Lato sensu é o método de curar as doenças por intermédio de seus agentes causais, manipulados mediante a técnica homeopática (dinamizados).

O termo nosódio foi criado por Constantine Hering para designar medicamentos produzidos a partir de produtos patológicos animais e vegetais (KossaK-RoMANACH, 2003). Os nosódios e os isoterápicos foram incorporados ao conceito de "bioterápicos", introduzido pela Farmacopeia Francesa (Amorim; Fontes, 2009), sendo usualmente utilizados como sinônimos.

Segundo Mitideiro (2002), no Brasil a isopatia é um recurso bastante utilizado na Medicina Veterinária pelos médicos veterinários homeopatas não só no tratamento de indivíduos, mas também no tratamento de rebanhos, sendo que a homeopatia vem crescendo em importância como uma alternativa na Medicina Veterinária e reconhecida pelo Conselho Federal de Medicina Veterinária (CFMV) a partir de 1995. Em 1999, ela foi reconhecida pelo Ministério da Agricultura para uso na agropecuária orgânica e no ano 2000 o CFMV regulamentou a concessão do título de especialista.
O uso de medicação homeopática no sal mineral pode ser uma alternativa para diminuir o uso de produtos químicos que, além de serem onerosos, determinam contaminaçôes ambientais, prejudicando toda a cadeia alimentar, e os indivíduos que manejam os produtos. Além do exposto, os medicamentos homeopáticos, ao diminuírem o número de aplicaçóes de quimioterápicos nos animais, reduzem a pressão de seleção sobre as populaçóes de carrapatos, retardando, assim, a seleção dessas populaçóes à resistência aos tratamentos convencionais (Arenales, 2006).

O uso de homeopatia já foi descrito por Signoretti et al. (2008) ao trabalharem com terneiros mestiços Gir $\mathrm{X}$ Holandês, no controle de endo e ectoparasitos, e por Silva et al. (2008) em novilhas da raça Purunã, no controle de Rhipicephalus (B.) microplus.

Diante do exposto, o presente trabalho tem como objetivo analisar a eficiência da utilização da isopatia como uma ferramenta de controle alternativo do carrapato bovino.

\section{MATERIAL E MÉTODOS}

\section{Preparação da isopatia}

A tintura-mãe e as formas farmacêuticas derivadas (diluições) foram produzidas de acordo com a Farmacopeia Homeopática Brasileira (ANvisa, 1997) para medicamentos de origem animal. A tintura-máe foi preparada por meio do processo de maceração utilizando-se como insumo inerte uma mistura de etanol, água e glicerina (1:1:1), no qual as larvas, que foram fragmentadas, ficaram em contato com quantidade suficiente de líquido extrator, em ambiente protegido da ação direta de luz e calor, e o recipiente foi agitado diariamente. Posteriormente foi filtrado, deixado em repouso por 48 horas e armazenado adequadamente. As formas farmacêuticas derivadas foram preparadas conforme o método de Hahnemann (Amorin et al., 2009) partindo da tintura-mãe até a potência $\mathrm{CH} 6$.

\section{Bovinos}

No experimento foram utilizadas 18 fêmeas bovinas de raça europeia (Devon x Red angus), devidamente identificadas, com brincos coloridos e numerados. Possuíam 24 meses de idade, as quais foram mantidas em um sistema de pecuária extensiva. Posteriormente, esses animais foram distribuídos em dois grupos com 9 bovinos, o controle (Lote A) e o tratado (Lote B) com isoterápico. 


\section{Área experimental}

O trabalho foi executado em uma propriedade de criação de gado de corte, situada no $2^{\circ}$ distrito do município de Canguçu, Rio Grande do Sul. Foram utilizados 2 potreiros de 9 hectares cada, com situação similar de vegetação e aguada, os quais foram roçados, e foi realizado diferimento por 100 dias (de janeiro a abril de 2011), com o intuito de zerar a infestaçáo da área por carrapatos.

\section{Introdução dos animais}

Em maio de 2011 os bovinos foram introduzidos nos potreiros para adaptação e observaçấo da ingesta de sal mineral, o qual seria utilizado como veículo para a administração da isopatia. Para confirmar a ausência de infestação por $R$. (B.) microplus, foram realizadas contagens de carrapatos quinzenalmente entre os meses de maio e julho, estando esses animais negativos.

\section{Administração da isopatia}

A partir de $1^{\circ}$ de agosto de 2011, o sal mineral foi ofertado ad libitum, sendo que o Lote A recebeu sal mineral com placebo (açúcar) e o Lote B sal mineral com isopatia.

\section{Infestação e contagem}

No final de dezembro de 2011, os 18 bovinos foram infestados artificialmente com 10 mil larvas de $R$. (B.) microplus. No $20^{\circ}, 21^{\circ}$ e $22^{\circ}$ dias após a infestaçáo foram realizadas contagens de fêmeas, acima de $4 \mathrm{~mm}$, nos 18 bovinos tanto do Lote A (controle), quanto do Lote B (tratado).

\section{Análise laboratorial}

Foram coletadas teleóginas (fêmeas com mais de $5 \mathrm{~mm}$ de comprimento) do Lote A e B, nos 3 dias de contagem $\left(20^{\circ}\right.$, $21^{\circ}$ e $22^{\circ}$ pós-infestação), as quais foram encaminhadas para o Laboratório de Doenças Parasitárias (LADOPAR) da Faculdade de Veterinária da Universidade Federal de Pelotas (UFPel).

No LADOPAR, as teleóginas de cada dia dos Lotes A e $\mathrm{B}$ foram divididas ao acaso em 9 grupos de 10 indivíduos, ou seja, foram incubadas 90 fêmeas ingurgitadas por dia, totalizando 270 indivíduos de cada lote.

Inicialmente as teleóginas foram lavadas em água corrente e secadas com papel toalha, para que fossem pesadas, fixadas em placas de Petri e incubadas em estufa à temperatura de $27^{\circ} \mathrm{C}( \pm 1)$ e umidade relativa de $80 \%( \pm 1)$. No $15^{\circ}$ dia de incubaçáo foi separada e pesada a massa de ovos férteis de cada placa e colocada em tubos de ensaio fechados com voal e identificados para posterior análise da eclodibilidade, a qual foi realizada no $30^{\circ}$ dia de incubação. Além disso, no $15^{\circ}$ dia foi realizada a pesagem das quenóginas. A partir desses dados foi avaliado o Índice de Eficácia Reprodutiva (IER) e o Índice Nutricional (IN), de acordo com BENNETT (1974), de cada grupo através das seguintes fórmulas:

$$
\begin{aligned}
& \text { IER }=\frac{\text { Massa de ovos X } 100}{\text { Massa das fêmeas }} \\
& \text { IN }=\frac{\text { Massa de ovos X 100 }}{\text { Massa das teleóginas }- \text { Massa das quenóginas }}
\end{aligned}
$$

\section{Análise estatística}

Para a comparação entre o número médio de teleóginas contadas do grupo controle e grupo tratado, e dos padróes biológicos, foi empregada a análise de variância (ANOVA) e o Teste LSD, através do programa Statistix 9.0.

\section{RESULTADOS E DISCUSSÃO}

Os animais do grupo tratado com a isopatia (Lote B) apresentaram uma reduçấo no número médio de teleóginas contatadas de $53,4 \%$, quando comparados com o grupo não tratado (Lote $\mathrm{A})(\mathrm{p}=0,001)$. Assim como constatado no estudo de Veríssimo (1988), que observou uma redução significativa $(\mathrm{p} \leq 0,05)$ na infestação por carrapatos em bovinos mestiços que receberam um bioterápico produzido a partir de teleóginas de $R$. (B.) microplus, dinamizado na $\mathrm{CH} 6$.

Discordando destes resultados, Signoretti et al. (2008) realizaram um trabalho administrando o Fator $\mathrm{C} \& \mathrm{MC}^{\oplus}$, que contém os isoterápicos $R$. (B.) microplus, Amblyomma cajennense, Haematobia irritans, Musca domestica, Bunostomum sp., Haemonchus contortus, H. placei, Nematodirus sp., Oesophagostomum sp., Strongyloides sp., Trichostrongylus axei, Trichostrongylus colubriformis, Trichuris sp., Eimeria sp., Cysticercus cellulosae, Dermatobia hominis, todos dinamizados na $\mathrm{CH} 12$, veículo sacarose q.s.p. 100 g, preparados de acordo com a Farmacopeia Homeopática Brasileira (Anvisa, 1997), e não observaram efeito na redução da infestação por $R$. (B.) microplus. Em contrapartida, esses mesmos autores (SignORETTI et al., 2010), ao trabalharem com fêmeas mestiças (Gir x Holandês), verificaram redução significativa na infestação por $R$. (B.) microplus, não sendo necessária a utilizaçáo de carrapaticida ao longo do experimento.

Concordando com os resultados do presente trabalho, Silva et al. (2008), utilizando o mesmo produto, observaram uma redução de 50,5\% no número médio de fêmeas 
ingurgitadas de $R$. (B.) microplus, quando comparado o grupo controle (sem tratamento) e grupo tratado com o princípio ativo Amitraz, ao longo de um ano de experimento.

Na Figura 1, observa-se que a adição do tratamento isoterápico ao sal mineral proporcionou um efeito positivo na redução de teleóginas contatadas no $20^{\circ}, 21^{\circ}$ e $22^{\circ}$ dias pós a infestação com larvas.

Verificou-se que no $20^{\circ}$ e $22^{\circ}$ dias a contagem de carrapatos do Lote $\mathrm{B}$ foi de 62,2 e $47,1 \%$, respectivamente, menor quando comparado com o Lote A $(\mathrm{p}<0,05)$. Já no $21^{\circ}$ dia, houve um decréscimo de $45,9 \%$, pós-infestação, sendo que o uso do isoterápico năo demonstrou diferença estatística significativa $(p>0,05)$ na contagem de carrapatos. Apesar de não haver diferença estatística significativa entre os grupos (tratado e controle), na contagem de carrapatos no $21^{\circ}$ dia pós-infestação, biologicamente essa diferença é expressiva, considerando que a capacidade de postura desse parasita (3.000 ovos/fêmea), assim a redução de 45,9\% no número médio de carrapatos, acarretará na diminuição da população no ambiente, consequentemente, reduzindo potencialmente a probabilidade de reinfestaçôes dos bovinos.

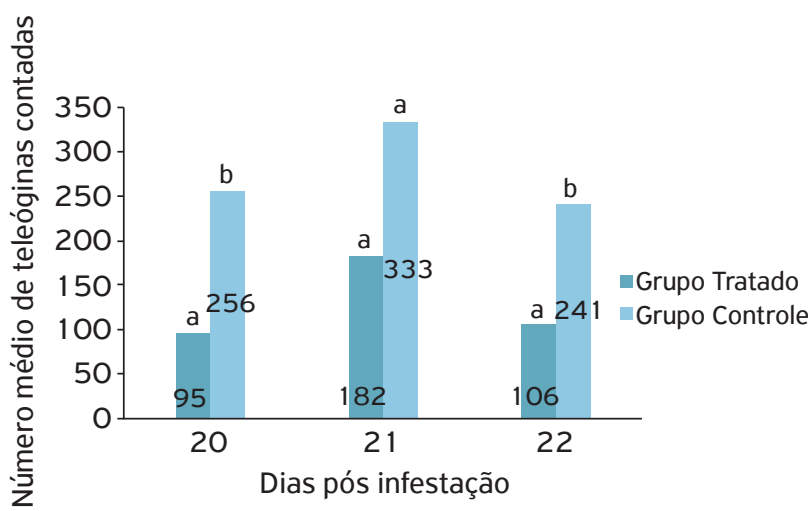

Letras diferentes sobre as colunas, para cada dia de contagem pósinfestação diferem estatisticamente entre si $(p<0,05)$.

Figura 1. Contagem de carrapatos no $20^{\circ}, 21^{\circ}$ e $22^{\circ}$ dia após infestação artificial com 10 mil larvas de $R$. (B.) microplus, em bovinos europeus criados em sistema extensivo, no município de Canguçu, Rio Grande do Sul.
Arenales et al. (2006) evidenciaram um efeito significativo de medicamentos homeopáticos no controle de $R$. (B.) microplus, atribuindo o adequado controle das endo e ectoparasitoses em bovinos, além do incremento de $37,9 \%$ no ganho de peso em relação aos animais não tratados, ao uso de complexo constituído de vários parasitos dinamizados.

As análises laboratoriais, para verificar a interferência do uso da isopatia sobre os parâmetros bióticos das teleóginas, podem ser observadas na Tabela 1 .

Conforme demonstrado na Tabela 1 , não houve diferença estatística significativa entre nos parâmetros biológicos dos Lotes A e B, o que também foi observado por Almeida et al. (2008) em experimento realizado com a utilização de nosódio (bioterápico) de Amblyomma cajannenses, no controle desse ectoparasita em coelhos. Entretanto, segundo Arenales (2002), que afirmou que após 7 a 10 dias alimentando bovinos tratados com isopatia, os carrapatos apresentaram dificuldade para se alimentar e começaram a murchar, até adquirirem um aspecto absolutamente em processo de mumificação; e o sangue das fêmeas ingurgitadas estará negro e coagulado. A autora afirmou ainda que teleóginas que caem nas pastagens realizam postura de uma massa de ovos amorfa, sem larvas viáveis, o que também não foi verificado no presente estudo. A redução dos parâmetros biológicos foi descrita por GAzim et al. (2010), que observaram uma interferência significativa do ciclo de carrapatos ingurgitados em bovinos tratados com bioterápicos (isoterápicos) em $\mathrm{CH} 12$ e CH30 produzidos a partir de uma matriz de fêmea de $R$. (B.) microplus ingurgitadas. Além disso, os testes in vitro mostraram redução de massa de teleóginas, redução da massa de ovos, redução da eclodibilidade e consequentemente redução da eficiência reprodutiva.

Apesar de não haver uma redução nos parâmetros biológicos entre o Lote $\mathrm{A}$ e $\mathrm{B}$, neste estudo, houve uma redução significativa na contagem de carrapatos no Lote B (tratado com isoterápico). Deve-se salientar que os resultados positivos obtidos no presente estudo podem estar relacionados ao modo de produção extensiva e ao manejo que foi realizado dentro deste contexto para proporcionar o bem-estar dos animais, evitando o

Tabela 1. Parâmetros biológicos de fêmeas ingurgitadas de $R$. (B.) microplus em bovinos sem a utilização de isopatia (Lote A) e tratado com isopatia (Lote $B$ ), em sistema de criação extensiva na região sul do Rio Grande do Sul.

\begin{tabular}{lccccc} 
Parâmetros biológicos & \multicolumn{2}{c}{ Lote A - Controle } & & \multicolumn{2}{c}{ Lote B - Tratado } \\
\cline { 2 - 6 } Massa das teleóginas (g) & Média de massa & Desvio padrão & Média de massa & Desvio padrão & Valor $\mathbf{p}^{*}$ \\
\hline Massa de ovos (g) & 0,2310 & 0,683 & 0,2375 & 0,676 & $>0,05$ \\
\hline Eclodibilidade (\%) & 0,1250 & 0,442 & 0,1243 & 0,439 & $>0,05$ \\
\hline Massa de quenóginas (g) & 89 & 11,323 & 90 & 12,495 & $>0,05$ \\
\hline IER & 0,0464 & 0,449 & 0,0533 & 1,015 & $>0,05$ \\
\hline IN & 53,199 & 4,329 & 51,303 & 6,094 & $>0,05$ \\
\hline
\end{tabular}

*nível de significância de 5\%; IER: Índice de Eficácia Reprodutiva (massa de ovos X 100/massa das teleóginas); IN: Índice Nutricional (massa de ovos X 100/massa das teleóginas - massa das quenóginas) 
estresse excessivo, visto que a homeopatia tem suas bases na energização de substâncias e no equilíbrio da energia vital dos seres. de Rhipicepalus (Boophilus) microplus é uma alternativa promissora para o controle desse ixodídeo.

\section{CONCLUSÕES}

O uso de isopatia foi capaz de reduzir a infestação de Rhipicepalus (Boophilus) microplus, em 53,4\%, quanto comparado ao controle. Assim, a isopatia produzida a partir de matriz de larvas

\section{AGRADECIMENTOS}

Ao Luís Fernando Farias da Silveira e família, parceiros da Empresa Rural Lagoa Azul, pelo auxílio e dedicação, o que tornou possível a execução deste trabalho.

| | | | | | | | | | | | | | | | | | | | | | | | | | | | | | | | | | | | | | | | | | | | | | | | | | | | | | | | | | | | | | | | | | | | | | | | | | | | | | | | | | | | | | | | | | | | | | | | | | | | | | | | | | | | | | | | | | | | | | | | | | | | | | | | | | | | | | | | | | | | | | | | | | | | | | | | | | | | | | | | | | | | | | | | | | | | | | | | | | | | | | | | | | | | | | | | | | | | | | | | | | | | | | | | | | | | | | | | | | | | REFERÊNCIAS

ALMEIDA, L.R.; CUNHA, N.C.; LISBÔA, R.S.; MADUREIRA, R.C.; RANGEL, C.P.; VIANA, E.B.; FONSECA, A.H. Parâmetros biológicos de fêmeas adultas Amblyomma cajennense alimentadas em coelhos tratados com bioterápico ultradiluído. Ciência Rural, v.38, n.5, p. 1476-1478, 2008.

AMORIM, V.O.; FONTES, O.L. Bioterápicos. In: FONTES, O.L., CÉSAR, A.T.C., CHAUD, M.V; TEIXEIRA, M.Z; KISHI, M.A; AMORIM, V.O. Farmácia homeopática: teoria e prática. 3a ed. Barueri: Manole; 2009. p.21 1-234.

ANVISA. AGÊNCIA NACIONAL DE VIGILÂNCIA SANITÁRIA. Farmacopéia homeopática brasileira. $2^{\mathrm{a}}$ ed. São Paulo: Ateneu; 1997.

ARENALES, M.C. Homeopatia em gado de corte. In: I CONFERÊNCIA VIRTUAL GLOBAL SOBRE PRODUÇÃO ORGÂNICA DE BOVINOS DE CORTE, Corumbá, MS, 2002, Anais. Embrapa Pantanal. Disponível em: <http://www.cpap.embrapa.br/ agencia/ congressovirtual/pdf/ portugues/ 02pt05.pdf $\geq$. Acesso em: 24 jul. 2013.

ARENALES, M.C.; MORAES, A.; MORAES, F. Evaluation of the use of homeopathic products for the control of parasites and weight in Indian cattle (nelore), in Brazil. In: WORLD BUIATRICS CONGRESS, 24. Nice, 2006. Anais Nice: European College of Bovine Health Management Science; 2006.

BENNETT, G.F. Oviposition of Boophilus microplus (Canestrini) (Acari: Ixodidae). II. Influence of temperature humidity and light. Acarologia, v.16, n.2, p.250-257, 1974.

FARIAS, N.A.R. Carrapato dos bovinos. In: RIET-CORREA, F.; SCHILD, A.LLEMOS, R.A.A.; BORGES, J.R.J. Doenças de ruminantes e equídeos. $3^{\text {a }}$ ed. Santa Maria: Palotti; 2007. v. II, Cap. 1, 719 p.

GAZIM, Z.C.; FERREIRA, F.B.P; SILVA, A.V.; BOLOGNESE, K.C.; MELIN, E; MESSA,V.; JESUS, R.A.; COUTINHO, C.A.; SILVA, L.C.M. Efficiency of tick biotherapic on the control of infestation by Rhipicephalus (Boophilus) microplus in Dutch dairy cows. International Journal of High Dilution Research, v.9, n.33, p.156-164, 2010.

GRISI, L.; MASSARD, C.L.; MOYA BORJA, G.E.; PEREIRA, J.B. Impacto econômico das principais ectoparasitoses em bovinos no Brasil. Hora Veterinária, v.125, p.8-10, 2002.

KOSSAK-ROMANACH A. Isoterapia. In: KOSSAK-ROMANACH A. Homeopatia em 1000 conceitos. São Paulo: Elcid; 2003. p.399-407.
MITIDEIRO, A.M.A. Potencial do uso de homeopatia, bioterápicos e fitoterapia como opção na bovinocultura leiteira: avaliação dos aspectos sanitários e de produção. 2002. 132f. Dissertação (Mestrado em Agrossistemas) - Universidade Federal de Santa Catarina, Santa Catarina, 2002.

OLIVO, C.J; AGNOLIN, C.A.; PARRA, C.L.C.; VOGEL, F.S.F.; RICHARDS, N.S.P.S.; DE PELLEGRINI, L.G.; WEBE, A.; PIVOTO, F.; ARAUJO, L. Efeito do óleo de eucalipto (Corymbia citriodora) no controle do carrapato bovino. Ciência Rural, v.43, n.2, p.331-337, 2013.

PAIM, F.; DE SOUZA, A.P.; BELLATO, V.; SARTOR, A.A. Controle seletivo do Rhipicephalus (Boophilus) microplus em bovinos criados em campo nativo, no município de Lages, Santa Catarina, Brasil. Revista Brasileira de Parasitologia Veterinária, v.20, n.1, p.13-16, 2011.

SIGNORETTI; R.D. VERÍSSIMO, C.J.; SOUZA, F.H.M.; GARCIA; T.S.; OLIVEIRA, E.M., SOUZA, K.G.; MOURÃO, G.B. Desempenho e infestação por parasitos em machos leiteiros suplementados com sal proteinado com ou sem os medicamentos homeopáticos. Revista Brasileira de Parasitologia Veterinária, v.17, n.1, p.40-44, 2008.

SIGNORETTI, R.D.; VERÍSSIMO, C.J.; SOUZA, F.H.M.; OLIVEIRA, E.M.; DIB, V. Aspectos produtivos e sanitários de vacas mestiças leiteiras tratadas com produtos homeopáticos. Arquivos do Instituto Biológico, v.77, n.4, p.625-633, 2010.

SILVA, N.L; MOLETTA, J.L.; MINHO, A.; FILIPPSEN, L. Uso de isoterápico no controle da infestação natural por Boophilus microplus em bovinos. In: CONGRESSO BRASILEIRO DE HOMEOPATIA VETERINÁRIA, Porto Alegre, RS. Anais Arquivos da AMVHB, v.3, n. 1, p. 40-42, 2007.

SILVA, N.L.; MOLETTA, J.L.; MINHO, A.; FILIPPSEN, L. Use of biotherapic in the control of natural infestation by Boophilus microplus: pilot study. International Journal of High Dilution Research, v.7, n.22, p.36-38, 2008.

SILVA FILHO, M.L.; SILVA, L.B.; FERNANDES, R.M.; LOPES, G.S. Efeito do extrato aquoso e etanólico do angico preto sobre larvas de Rhipicephalus (Boophilus) microplus. Arquivo Brasileiro de Medicina Veterinária e Zootecnia, v.65, n.3, p.637-644, 2013.

VERÍSSIMO, C.J. Utilização do nosódio Carrapatinum em bovinos sensíveis ao carrapato Boophilus microplus (Canestrini). Pesquisa Homeopática, v.5, n. 1, p.21-25, 1988. 